\title{
(2) OPEN ACCESS \\ Non-static framework for understanding adaptive designs: an ethical justification in paediatric trials
}

\author{
Michael OS Afolabi, ${ }^{1}$ Lauren E Kelly ${ }^{1,2,3}$
}

'Department of Pediatrics and Child Health, Faculty of Health Sciences, University of Manitoba, Winnipeg, Manitoba, Canada

${ }^{2}$ Children's Hospital Research Institute of Manitoba, Winnipeg, Manitoba, Canada

${ }^{3}$ George \& Fay Yee Centre for Healthcare Innovation, Winnipeg, Manitoba, Canada

\section{Correspondence to} Dr Michael OS Afolabi, Department of Pediatrics and Child Health, University of

Manitoba, Winnipeg, Manitoba MB R3T 2N2, Canada; michael.afolabi@umanitoba.ca; curiousmaik|@yahoo.com

Received 18 January 2021 Accepted 25 June 2021

\section{Check for updates}

(C) Author(s) (or their employer(s)) 2021. Re-use permitted under CC BY-NC. No commercial re-use. See rights and permissions. Published by BMJ.

To cite: Afolabi MOS, Kelly LE. J Med Ethics Epub ahead of print: [please include Day Month Year]. doi:10.1136/

medethics-2021-107263

\section{ABSTRACT}

Many drugs used in paediatric medicine are off-label. There is a rising call for the use of adaptive clinical trial designs (ADs) in responding to the need for safe and effective drugs given their potential to offer efficiency and cost-effective benefits compared with traditional clinical trials. ADs have a strong appeal in paediatric clinical trials given the small number of available participants, limited understanding of age-related variability and the desire to limit exposure to futile or unsafe interventions. Although the ethical value of adaptive trials has increasingly come under scrutiny, there is a paucity of literature on the ethical dilemmas that may be associated with paediatric adaptive designs (PADs). This paper highlights some of these ethical concerns around safety, scientific/social value and caregiver/guardian comprehension of the trial design. Against this background, the paper develops a non-static conceptual lens for understanding PADs. It shows that ADs are epistemically open and reduce some of the knowledge-associated uncertainties inherent in clinical trials as well as fast-track the time to draw conclusions about the value of evaluated drugs/ treatments. On this note, the authors argue that PADs are ethically justifiable given they (1) have multiple layers of safety, exposing enrolled children to lesser potential risks, (2) create social/scientific value generally and for paediatric populations in particular, (3) specifically foster the flourishing of paediatric populations and (4) can significantly improve paediatric trial efficiency when properly designed and implemented. However, because PADs are relatively new and their regulatory, ethical and logistical characteristics are yet to be clarified in some jurisdictions, the cooperation of various public and private stakeholders is required to ensure that the interests of children, their caregivers and parents/ guardians are best served while exposing paediatric research subjects to the most minimal of risks when they are enrolled in paediatric trials that use ADs.

\section{INTRODUCTION}

Traditional clinical trials have fixed designs with predefined sample sizes, allow data analysis at the end of any given study and leave minimal room for modifications of the planned trial. In contrast, a non-traditional class of study design known as adaptive designs (ADs), including basket trials, platform trials, umbrella trials, drop the loser, play the winner, seamless phase II/III ${ }^{1}$ or response adaptive randomisations, allow preplanned modifications on the basis of the data accumulating over the course of a trial. ${ }^{2}$ Clinical trials that use ADs may be useful in the accelerated provision of safe and effective drugs as they offer efficient and potentially cost-effective benefits to how trials are traditionally designed and conducted, ${ }^{3}$ without undermining study validity and integrity. ${ }^{4}$ In paediatric practice, many medicines are off-label ${ }^{5}$ and pharmacological data are often extrapolated from adults to children, though there are metabolic and developmental differences between both groups ${ }^{6}$ highlighting a need for efficient clinical trials.

The use of off-label drugs, compared with drugs with population-specific indications, are associated with more adverse drug reactions ${ }^{78}$ as well as underdosing and overdosing in children, which underscores the paediatric population's need for quality, safe and efficacious drugs formulated for children, infants and neonates. ${ }^{59} 10$ Traditional clinical trials often answer a single specific question with a predetermined sample size (informed by data gathered mostly from other populations) presenting challenges for rare diseases or diseases that are only present in childhood, with limited data about disease course and variability in response across different age groups. Given the costs of conducting clinical trials and many areas in need of study, there is an urgent need for alternative trial designs such as ADs in paediatric clinical trials.

Whereas there is a rising call for the incorporation of $\mathrm{AD}$ frameworks to increase the efficiency of clinical trials, ${ }^{11}{ }^{12}$ their ethical value has increasingly come under scrutiny. Although a limited amount of extant literature has engaged the ethical concerns that are specific to outcome-adaptive studies, ${ }^{13}$ there is a paucity of literature on the ethical challenges that may be specifically associated with paediatric adaptive designs (PADs). Against this background, this paper identifies and discusses some of the central ethical dilemmas that PADs generate. It uses a non-static conceptual lens to understand ADs in general and PADs in particular, ultimately arguing that PADs are justifiable on pragmatic and ethical grounds due to their multiple layers of safety, social/ scientific value and their potential to meet the unique drug needs of paediatric populations.

\section{ADAPTIVE DESIGNS IN CLINICAL TRIALS}

ADs incorporate prospectively planned modifications of one or more specific aspects of the study design and/or the associated hypotheses, using data (from subjects in the study and occasionally from other studies being conducted in parallel) accumulated in the course of a clinical trial. ${ }^{10}$ Such adaptations take several forms and are always prespecified. For instance, the subjects may be initially allocated to the experimental or standard treatment arm in a fixed 1:1 ratio, but the allocation ratio may be altered to tilt towards the preferred or better arm ('play the winner') ${ }^{14}$ if the emerging data favour 
one of the treatment arms. ${ }^{15}$ Other changes associated with ADs include trial eligibility criteria, dosing, sample size re-estimation to enhance power, the statistical analysis plan, the choice of the primary outcome, outcome adaptive randomisation to allocate more participants to the potentially superior treatment arm and ending the trial if an interim analysis indicates a low probability of detecting a difference between the treatment and control. ${ }^{116}$

Compared with fixed designs, ADs generally use smaller sample sizes for the same statistical power and can address a research question in more detail. Based on modelling, it has been estimated that up to a $60 \%$ reduction in sample size may be achieved with ADs compared with traditional clinical trials that incorporate rigid sample sizes. ${ }^{17}$ However, some research questions may require an increased sample size to provide the relevant answer. Sample size modifications potentially offer improved information on how specific interventions may be applied, for instance, in the context of dosing or a subgroup. ${ }^{18}$ Such information should, therefore, lead to an earlier identification of ineffective therapies compared with traditional clinical trials, thereby cutting down the overall participant burden and cost of a trial. ${ }^{19}$

Regardless of these potential advantages, some scholars argue that $\mathrm{ADs}$ foster some degree of injustice, ${ }^{13}$ may add to the burden borne by some of the participants enrolled in such trials, ${ }^{20}$ complicate the process of consent ${ }^{21}$ and may increase how patients fail to distinguish between being under clinical care and clinical research (therapeutic misconception). ${ }^{22}$

\section{ETHICAL CHALLENGES OF ADAPTIVE DESIGNS IN PAEDIATRIC CLINICAL TRIALS}

Although the adaptations that will be made in trials that use ADs are prespecified, they are not uniform across all trials. That is, while adaptive trial A may prespecify accruing data-based modifications in randomisation ratios, an adaptive trial B may specify dropping an inferior treatment arm. ${ }^{23}$ This raises the possibility that the ethical issues that specific adaptations in individual trials may vary. However, from a clinical research perspective, the central ethical quandaries surrounding the use of ADs in paediatric clinical trials largely revolve around issues of safety, scientific/social value and comprehension of the trial design by parents/caregivers as well as children and youth participants. A closer examination of the nature and nuances surrounding these issues follows.

\section{Safety issues in paediatric adaptive designs}

Although children can benefit greatly from clinical research, the added protection they often need (such as closer clinical monitoring), perceived barriers and lack of resources/infrastructure to incentivise multisite collaboration minimise their participation in clinical trials. ${ }^{24}$ This scenario generally exposes them to off-label drugs and unauthorised therapies due to a paucity of evidence generated through paediatric clinical trials and submission for marketing authorisations. ${ }^{25}$ This state of affairs has, however, led to untoward consequences in the past. For instance, the use of chloramphenicol for neonatal sepsis and rapid infusion of concentrated bicarbonate to correct metabolic acidosis were associated with increased mortality and morbidity in critically ill neonates. ${ }^{26}$ Whereas this observation underscores the importance of clinical trials in neonates, it does not legitimise conducting paediatric trials at the expense of their safety. Indeed, safety in PADs should minimise risks varying from known or possible therapeutic-related harms to indignities, in incorporating safety data from preclinical models and other populations.

When the safety and efficacy of experimental therapies are unproven, withholding them from some potentially eligible patients may be ethically justifiable ${ }^{25}$. However, safety concerns arise in the context of ADs that employ an adaptive randomisation technique because some of the participants will not receive the experimental therapy with promising results (based on accruing data). Rather, the trial will continue to assign some participants (though limited) to a treatment for which there is an increasing statistical probability that it will be inferior. ${ }^{13}$ At first glance, it would seem that this is especially problematic in the context of PADs due to the involvement of children. In other words, being aware of the relative superiority of one treatment over the other (or others, depending on how many arms are incorporated into the specific trial) and continuing to assign some children to a treatment that seems to be relatively less effective seems problematic. Indeed, if safety is contextualised to mean positive in-trial clinical benefits with attendant minimal or no side-effects, exposing such limited number of children to a potentially inferior treatment would imply a lesser degree of safety. However, this potential 'pharmacological inferiority' normally occurs in traditional trials with a fixed sample size but is hardly evident until the end of recruitment and data analysis. In other words, the preplanned modifications that occur in PADs decrease the number of those that would be allocated to a potential in-trial unintended 'harm'. Such possible unintended harms are, however, intrinsic to clinical trials and are not a unique feature of adaptive designs.

The continued allocation to a probable 'inferiority arm' of trials that are adaptive may, however, serve useful epistemic and pragmatic purposes. For example, it could serve as a means of further learning about any delayed treatment effects or effects that may be due to temporal trends. The possibility that temporal trends lead to changes in the dynamics between inferiority and superiority arms supports the idea that keeping all arms running in PADs may improve the quality of the conclusions that will be drawn from data generated in such trials. This perspective is reinforced by the observation that toxicity, efficacy and delayed effects in clinical trials do not always occur within the same time-frame. $^{27}$

PADs also tend to have a uniquely better safety net compared with traditional paediatric clinical trials because data related to efficacy are likely to accrue faster. If this leads to dropping a treatment arm where efficacy is highly improbable (eg, in a multiarm trial) ${ }^{28}$ or excluding some individual participants (eg, based on specific biomarkers evaluated during an interim analysis), ${ }^{23}$ then paediatric participants will be spared unwarranted exposure to the given drug under investigation. However, like traditional trials, in-trial safety issues that derive directly from the use of adaptive designs in clinical trials have hardly been addressed by extant regulatory guidelines and are often left at the discretion of individual sponsors and trialists. In the UK, paediatric research is still generally expected to involve no greater than minimal or low risk. ${ }^{29}$ In North America, the situation is roughly the same as there is no compensatory mechanism for research-related harms that paediatric participants may experience. ${ }^{22}$ This has prompted Fleischman and Collogan to argue that there is a need for a government-sponsored regulation to develop a no-fault insurance pool to meet this need. ${ }^{22}$ In the context of PADs, such a regulatory reform is more pressing and highlights the need for regulators, trialists, funders and researchers to collaboratively come up with a viable way to specifically address in-trial-related adverse reactions in PADs. This will help to foster trust in the 
trial community, which is integral to achieving the full potential of clinical trials that use adaptive designs. ${ }^{30}$

Last, if the predominant practice of using off-label and unlicensed drugs constitutes a paediatric safety issue due to the associated increased risks of adverse drug reactions, ${ }^{31}$ the use of PADs to specifically evaluate drug safety, posology and efficacy in the paediatric population is one of the options available to address this safety concern as well as generate drug data that are specific to the paediatric patient population.

\section{Scientific/social value of paediatric adaptive designs}

Paediatric clinical trials are necessary as certain disease conditions and drug responses are children-specific, ${ }^{6}$ though research studies may not offer direct benefit to participants and may expose them to risks of potential harm. ${ }^{32}$ In other words, paediatric trials including those that use AD-based frameworks specifically seek to create scientific and social value to paediatric populations through identification and optimisation of safe and effective therapies, dosing regimens as well as the faster rejection of treatments/drugs that are ineffective or toxic. Indeed, the rhetoric of social value is not just an ethical benchmark for Western-based research, ${ }^{33}$ but extends to contexts where nonwestern drugs and phytomedicines are being evaluated via clinical trials. ${ }^{34}$

In the context of clinical research, methods that minimise the allocation of research participants to an inferior treatment are ethically appealling ${ }^{22}$ and aligns, prima facie, with the moral principle that requires physician investigators to maximise benefit (beneficence) and reduce harm (non-maleficence). ${ }^{13} \mathrm{Harm}$ reduction has been previously reported in PADs. For example, the adaptive design used in the PREMILOC study enabled the researchers to end the trial as soon as efficacy was established based on accruing data, thereby preventing the further allocation of neonates to the placebo arm of the study. ${ }^{35}$ This re-echoes the notion that PADs reduce the exposure of in-trial participants to ineffective therapies. It likewise suggests the faster time to answer research questions with PADs, if interpreted and communicated wisely, with the potential to implement successful treatments more quickly into paediatric practice. Applied to the context of rare childhood diseases like primary ciliary dyskinesia and Duchenne muscular dystrophy where faster access to early interventions and treatments are critical to reduce morbidity, ${ }^{36}$ the conclusions of paediatric adaptive trials will have more immediate and long-term clinical benefits. This will ultimately improve paediatric trial efficiency by significantly shortening the time it will take for paediatric medicines (that are successfully evaluated via PADs) to enter into clinical use. The attendant implication of this is that children will generally not have to wait for several years for trial data to filter into paediatric practice. ${ }^{37}$

\section{Participant and caregiver comprehension of paediatric adaptive designs}

The ethical requirement that participants understand trials prior to providing consent implies that inadequate information will stifle meaningful choice, constrain autonomy ${ }^{38}$ and may invalidate the ethical force of any trial consent given under a state of ignorance. ADs differ from traditional clinical trials and have an increased likelihood of complexity; ${ }^{39}$ as such, there is a possibility that caregivers and parents/guardians may have a hard time comprehending the changing dynamics of the specific PAD-based trial for which their consent is required. PADs, however, also elicit specific questions about assent, the capacity of children to 'positively agree' to participate in a trial/study. Assent in research is not confined to the sphere of ensuring that the regulatory box is properly checked off. ${ }^{40}$ Its importance centres around reducing possible physical/psychological harm, according respect to the child as a human being, and his/her universal rights. ${ }^{41}$

Although the default attitude is that assent should only be sought from children who are able to understand trials and other research studies, ${ }^{42}$ the complexity of PADs raises the possibility that valid assent may be fraught with difficulties in the context of paediatric research that use adaptive trial designs. Such a viewpoint is reinforced by the inadequacy of disclosure and comprehension in the context of trials based on ADs. ${ }^{21}$ Taken together, an ethically problematic implication of these strings of thought is that some caregivers and many of the children participants may not fully understand the what and what-not that PADs entail generally and the particular dynamics and nuances of the specific adaptive-based paediatric trial where their participation is needed. However, the challenge of understanding inherent in PADs for the purpose of ethically valid parental consent and assent of eligible children could potentially be resolved through transparancy, meaningful engagement with a trained research coordinator, the use of visual aids to enhance comprehension and an evaluation of information obtained during the consent process. In this regard, specialised comic books ${ }^{43}$ may be suited for younger children while other tools such as the modified MacArthur Competence Assessment Tool for Clinical Research may be suitable for older children. ${ }^{44}$ Whereas engagement with children, youth and parents in the design process may ensure consent and assent forms convey clear and concise information on study design, empirical research and further bioethical reflection on the process of informed consent and assent in PAD are important knowledge gaps that require scholarly investigation.

Using age-based criteria, some commentators argue that assent may be sought from children from age 7 onwards. ${ }^{42}$ Applied to the context of PADs, this implies that only parental/caregiver consent will be ethically essential when younger-than-7-year-old children are enrolled in clinical trials. However, in addition to parental/caregiver consent, children who fall between the 8 and 18 age spectrum will need to provide assent. Yet, there is a possibility that some younger-than-7-year-old children whose parents/care-givers consent to their participation may choose not to participate in some PADs. In such a scenario, the principle of dissent should apply. Put simply, this will involve the bodily reaction to a specific trial in a way that conveys any form of distress. ${ }^{45}$ Finally, there is the possibility that some children who previously gave assent to a PAD-based clinical trial prior to the age of 18 will turn the legal age before the end of that trial. In such scenarios, the ethical validity of those PADs will depend on the respective youth participants to give their consent for continued participation in the trial (without the need for parental consent). They should also have the liberty to decline continued participation without any consequences.

\section{A NON-STATIC CONCEPTUALISATION OF PAEDIATRIC ADAPTIVE DESIGNS}

Although certain ethical concerns may arise in the context of PADs, such concerns may be reconciled if adaptive designs, of which PADs are a specific subset, are conceptualised as an open way of engaging the epistemic and practical complexities surrounding clinical trials. According to Karl Popper, the world is emergent and requires non-static explanatory approximations of its realities. ${ }^{46}$ In epistemological terms, this implies that knowledge often evolves and adapts to an equally evolving reality. Seen in this light, a non-static way of describing PADs is that they constitute epistemically open and fluid ways of conducting 
paediatric research by specifically allowing investigators to use developing data to make pragmatic decisions in the best interest of science and the children and young adults who participate in paediatric clinical trials.

An important implication of this non-static perspective of PADs is that it may reduce some of the knowledge-associated uncertainties inherent in clinical trials by reflecting real-world, population-specific shifts in knowledge. Since uncertainty, the absence of certitude or exact knowledge ${ }^{47}$ can only be diminished via knowledge acquisition; ${ }^{48}$ conceptualising PADs via a non-static prism reflects how real-world changes occur in the course of knowledge acquisition vis-à-vis eliminating some of the uncertainties tied to trial drugs and other interventions in paediatric medicine. This remark ties in with Alex London's argument that ADs reflect how changes occur in an idealised learning health system in response to reliable evidence about the relative strengths of specific treatment modalities. ${ }^{49}$ In other words, PADs seek to engage a shifting or non-static state of affairs in relation to eliciting therapeutic information, solving therapeutic uncertainties about investigational compounds/ drugs and ultimately drawing conclusions about the efficacy or inefficacy of trial drugs.

It is important to note that decisions in real life are made in such a way that newer information often informs decisional shifts. For instance, imagine that person A needs a good protein-rich cereal and finds that products $\mathrm{X}, \mathrm{Y}$ and $\mathrm{Z}$ are available options. After a period of interchanging the consumption of each, if person A comes to know (eg, based on finding out and comparing the nutritional components of each brand) and believe that product $\mathrm{X}$ is nutritionally superior to $\mathrm{Y}$ and $\mathrm{Z}$, the subsequent practical implication will be that person A will buy more of $\mathrm{X}$ and less (if any) of $\mathrm{Y}$ and $\mathrm{Z}$. In other words, unless there is another compelling reason (driven by hitherto unavailable information), most rational persons in person A's shoes will not abandon their consumption of X. Transposed to the context of clinical trials, PADs may be said to mirror the on-the-go decisional refinements that govern our daily human experiences but use the constraints of predefined rules for making refinements without compromising methodological rigour in clinical trial designs. This last remark, however, needs further elaboration. Unlike the analogy described, PADs employ rigorous statistical tools rather than only apparent efficacy, inefficacy or toxicity to drive interim decisions and the conclusions reached at the end of the trial. Specifically, PADs as subsets of ADs incorporate preplanned statistical analysis plans that can help control operational biases and type 1 errors. ${ }^{23}$ Biostatisticians are also working on other ways to control type 1 errors. For instance, the Bonferroni-Holm test procedure based on conditional error rates of individual treatment-control comparisons. ${ }^{50}$

Although the class of adaptive designs is broad to the extent that the methodological and statistical challenges (and solutions) are individually determined, ${ }^{51}$ critics often overlook this and paint such challenges with a broad brush. ${ }^{52}$ For instance, within the statistical community, there is a schism about the benefits of using either the frequentist or Bayesian approach in ADs that use response-adaptive randomisation, ${ }^{53}$ some of which are tied to the reliability of the $\mathrm{p}$ values of the evaluated parameters. ${ }^{23}$ Although rigorous statistical tools are used to ensure the statistical integrity of ADs including the use of random stratification and blocked randomisation, ${ }^{54}$ some biostatisticians have increasingly argued that the $\mathrm{p}$ value is not the sole indicator of plausibility, truth or presence of an association or effect. ${ }^{55}$ In other words, statistical inference is not the sole arbiter of scientific inference. Increasingly, some biostatisticians are questioning the gold standard status of the $\mathrm{p}$ value,${ }^{5657}$ arguing that statistical inferences could be strengthened if decisions based on $p$ values were replaced by a biological evaluation of effect sizes and their CIs, which can provide insights about the precision of observed effects. $^{58}$

\section{Justifying paediatric adaptive trials}

The notion of pragmatic justification entails justifying moral norms on the basis of their capacities to achieve some of the goals of morality. ${ }^{38}$ Morality, however, entails two important dimensions: the experiential or practical aspect of the moral life and the ethical ideas that govern the lived experiences. As such, a robust justification should address both of these aspects of morality. In relation to adaptive designs, there are pragmatic and ethical arguments that may justify PADs.

\section{Pragmatic justification of paediatric adaptive trials}

As previously pointed out, there are some potential safety issues inherent in all clinical trials including those with PADs. However, it is important to note that some degrees of uncertainty (for instance, about possible treatment outcomes and side effects) are an inevitable aspect of contemporary biomedical/clinical research, and critical components of equipoise are needed to justify that a clinical trial is necessary. If the potential for harm constitutes an almost unavoidable aspect of most clinical research, the practical response will be ensuring that such harms are kept to the barest minimum as much as possible.$^{59}$ PADs meet this principle because they have multiple layers of safety. For instance, their generally small sample size ${ }^{17}$ and use of accrued data imply that a minimal number of children enrolled will be exposed to ineffective treatments. ${ }^{60}$ In addition, the quick turnaround time of $\mathrm{PADs}^{5}$ implies that responder paediatric populations will be identified in a timely manner. ${ }^{61}$ This statistically cuts the potential risk of undue exposure to test drugs. It also raises the theoretical possibility that PADs may provide useful data about hitherto uncertainties related to specific trial drugs, devices and vaccines. The ability to add new treatment arms as evidence arises in some PADs are particularly valuable, especially in the context of the unknown, for example, during a pandemic. It has been reported that clinical trial designs that use frequent interim analyses via early stopping rules outperform those with a single end-of-study analysis in the average time to conclusion, average sample size as well as the probability of drawing viable conclusions. ${ }^{62}$ This reinforces the pragmatic value of PADs.

The appeal of the pragmatic justification of the framework of PADs developed in this article is further underscored by the fact that the use of real-world evidence has not been fully developed in paediatrics. ${ }^{63}$ Therefore, PADs create social/scientific value for paediatric populations by being responsive to their unique pharmacotherapeutic needs. If a part of the goals of innovative therapies is the provision of evidence of benefit and elucidation about the level of consequent risk, ${ }^{25}$ then PADs may be considered as an important tool for evaluating therapeutic benefits and clarifying their attendant risk dynamics in the paediatric population.

\section{Ethical justification of paediatric adaptive trials}

Ethics entails the possibilities of rationally founding and justifying our human conduct. ${ }^{64}$ Since human flourishing remains a central focus of societal telos, ${ }^{65}$ and this end aligns with the goal of the contemporary bioethical enterprise as articulated by Van Rensselaer Potter, ${ }^{66}$ ethically justifying PADs will involve showing how paediatric clinical trials that use adaptive designs will foster human flourishing in general and the flourishing of paediatric populations in particular. In paediatric practice, the 
off-label use of established adult medicines is rife, resulting in increased adverse events and suboptimal efficacy in the target population. ${ }^{67}$ If justice involves preventing an inequitable burden of research, ${ }^{68}$ then justice in the context of PADs will entail children bearing the burden of paediatric-specific trials. However, this could be after novel therapies have been previously evaluated in animal and adult human populations ${ }^{69}$ in order to minimise possible harms in neonates, infants, children and adolescents.

Although some commentators have argued that ADs (and by implication PADs) potentially have biases, ${ }^{39}$ taking decisions about increasing or decreasing allocation to treatment/ control arms independently of the treating physicians by using predefined, statistically justified definitions for superiority and futility can help minimise such subjective biases. The use of intense and thoughtful planning with $\mathrm{AD}$ experts during the design phase ${ }^{14}$ of a PAD can also help minimise some of the methodological biases/concerns.

Finally, Marianna Kruger has advocated that it is the duty of paediatric clinicians to assist in ensuring improved access to efficacious and safe medicines for children. ${ }^{24}$ However, access to safe and efficacious medicine for children to meet their specific disease burdens (eg, for Kawasaki disease, necrotising enterocolitis and Duchenne muscular dystrophy) and the physiological impact of development cannot be realised if novel and promising trial designs such as PADs are not explored. In other words, whereas children are vulnerable members of the society for whom utmost protection is necessary ${ }^{70}$ allowing them to participate in PADs is a promising means of protecting them from continued exposure to unregistered drugs and adult-based extrapolated dosing regimens.

Another important theme is the question of clinical equipoise. Although there are clear distinctions between the ends of receiving in-trial treatment and participating in one of the arms of a clinical trial, a number of bioethicists consider clinical equipoise-a state of uncertainty about 'superior' efficacy among the treatments being evaluated in a given trial一as an integral ethical principle governing the rationale for a clinical trial. ${ }^{71}$ However, bioethicists still disagree about this requirement.$^{72-74}$ The concept of equipoise has not been widely incorporated into paediatric research due to several areas of poor evidence on effectiveness and safety of paediatric therapeutic interventions that are based on adult-extrapolated data $\cdot{ }^{75}$ Hence, the ethical ramifications it elicits in paediatric clinical trials have hardly received any systematic engagement. For example, the Canadian Tri-Council Policy on research involving humans is silent on the issue of clinical equipoise in paediatric research. ${ }^{76}$

However, the specific nature of a given paediatric trial, the treatment arms that are being compared and the specific preplanned modifications will ultimately determine whether or not the principle of equipoise holds. In the context of a PAD that uses a response-adaptive or a biomarker-driven randomisation technique, if the accruing data elicit an adaptation such that more enrolled patients are allocated to intervention B and less to $\mathrm{A}$, the state of equipoise will appear to have been violated. However, because clinicians and other clinical researchers who run PADs would not explicitly know this (due to blinding) as only the Data Monitoring Committees (DMCs) usually have access to the evolving data at interim analyses, clinical equipoise essentially remains unbroken. Also, the possibility that temporal trends and delayed drug effects may alter the eventual superiority-inferiority arm outcome of PADs implies that even the DMCs remain in varying states of uncertainty. If the adaptive decision required in an ongoing PAD is dose-related, the same scenario is applicable and equipoise can be maintained with appropriate blinding. However, in a PAD where pharmacokinetic differences are being evaluated, ${ }^{77}$ the need to satisfy the principle of clinical equipoise will not arise because a state of efficacy-related uncertainty is not central to such trials. This again reinforces the idea that the specific dimensions of the ethical quandaries that PADs will elicit will be tied to the preplanned modifications in each trial.

\section{CONCLUSION}

The prevalent use of off-label drugs and extrapolated data of drugs meant for the adult population in paediatrics is ethically worrisome due to the potential for adverse drug reactions and possible undertreatment and overtreatment. This calls for increased conduct and funding of paediatric clinical trials, which incorporate innovative and efficient methodological designs such as adaptive designs. However, such PADs must be ethically viable and justifiable. ${ }^{31}$ Using a non-static explanatory framework, this paper has argued that paediatric adaptive designs may be justified on ethical and pragmatic grounds. However, because PADs are relatively new and their regulatory, ethical and logistical considerations are yet to be fully clarified ${ }^{5}$ the cooperation of various public and private stakeholders such as clinicians, parents, researchers and regulatory bodies ${ }^{7879}$ will be integral to clarifying and debating their ethical, statistical and operational issues.

Such a process can help produce consensual guidelines on how to generally engage the issues raised by the complexities of PADs including the fluid responsibilities of trialists, sponsors, parental consent and paediatric assent/dissent. This should ultimately ensure that the interests of children are best served while exposing them to minimal risks and building an evidence base for optimal pharmacotherapy. Without exploring promising alternative trial designs such as PADs, the likelihood of improving healthcare for minors now and in the future may lag behind. ${ }^{80}$ However, such designs require additional transparency, communication and reporting to enhance the credibility and interpretability of the results. ${ }^{81}$ Finally, one of the principal goals of ADs and by implication PADs is fast-tracking the clinical development process while minimising the number of patients that will be exposed to experimental treatments via early termination of a trial for futility, efficacy or toxicity. ${ }^{23}$ As such, if PADs are properly carried out with the right statistical measures to ensure the statistical as well as scientific integrity of the conclusions, and if the trial-specific quandaries raised by the trial protocol are properly engaged; then they are one of the ethically appealing options that can improve the efficiency of paediatric clinical trials.

Correction notice This paper has been updated to amend order of references 62 and 63 .

Contributors MA conceptualised and wrote the draft manuscript. LK made various technical inputs as the manuscript evolved. Both authors participated in the review and finalization of the manuscript.

Funding This work is funded through a Research Manitoba New Investigator Operating Grant. Grant number is \#3642.

Competing interests None declared.

Patient consent for publication Not required.

Provenance and peer review Not commissioned; externally peer reviewed.

Data availability statement Data sharing not applicable as no datasets generated and/or analysed for this study. Not applicable.

Open access This is an open access article distributed in accordance with the Creative Commons Attribution Non Commercial (CC BY-NC 4.0) license, which 
permits others to distribute, remix, adapt, build upon this work non-commercially, and license their derivative works on different terms, provided the original work is properly cited, appropriate credit is given, any changes made indicated, and the use is non-commercial. See: http://creativecommons.org/licenses/by-nc/4.0/.

\section{REFERENCES}

1 Bretz F, Koenig F, Brannath W, et al. Adaptive designs for confirmatory clinical trials. Stat Med 2009;28(8):1181-217.

2 Robertson DS, Lee KM, Lopez-Kolkovska BC. Response-adaptive randomization in clinical trials: from myths to practical considerations. arXiv preprint 2020.

3 Patel S, Steen D. Widening the path and window of opportunity for FDA approval of non-vitamin K oral anticoagulant specific antidotes and reversal agents. J Thromb Thrombolysis 2016;41(2):285-92.

4 Chang $\mathrm{M}$, Chow S-C, Pong A. Adaptive design in clinical research: issues, opportunities, and recommendations. J Biopharm Stat 2006;16(3):299-309.

5 Kelly LE, Dyson MP, Butcher NJ, et al. Considerations for adaptive design in pediatric clinical trials: study protocol for a systematic review, mixed-methods study, and integrated knowledge translation plan. Trials 2018;19(1):1-9.

6 Edwards SD, McNamee MJ. Ethical concerns regarding guidelines for the conduct of clinical research on children. J Med Ethics 2005;31(6):351-4.

7 Magalhães J, Rodrigues AT, Roque F, et al. Use of off-label and unlicenced drugs in hospitalised paediatric patients: a systematic review. Eur I Clin Pharmacol 2015;71(1):1-13.

8 Ku LC, Smith PB. Dosing in neonates: special considerations in physiology and trial design. Pediatr Res 2015;77(1-1):2-9.

9 Kimland E, Odlind V. Off-Label drug use in pediatric patients. Clin Pharmacol Ther 2012;91(5):796-801.

10 Food and Drug Administration. Guidance for industry adaptive design clinical trials for drugs and biologics, 2018. Available: https://www.fda.gov/downloads/drugs/ guidances/ucm201790.pdf [Accessed 27 Jun 2020].

11 Rosenberger WF, Sverdlov O, Hu F. Adaptive randomization for clinical trials. J Biopharm Stat 2012;22(4):719-36.

12 Kairalla JA, Coffey CS, Thomann MA, et al. Adaptive trial designs: a review of barriers and opportunities. Trials 2012;13(1):1-9.

13 Saxman SB. Ethical considerations for outcome-adaptive trial designs: a clinical researcher's perspective. Bioethics 2015;29(2):59-65.

14 Rosenberger WF. Randomized play-the-winner clinical trials: review and recommendations. Control Clin Trials 1999:20(4):328-42.

15 Joffe S, Truog R. Equipoise and randomization. In: Emanuel EJ, ed. The Oxford textbook of clinical research ethics. Oxford University Press, 2008: 245-60.

16 Chow S-C, Chang M. Adaptive design methods in clinical trials - a review. Orphanet Rare Dis 2008;3(1).

17 van der Lee JH, Wesseling J, Tanck MW, et al. Sequential design with boundaries approach in pediatric intervention research reduces sample size. J Clin Epidemiol 2010;63(1):19-27.

18 Hey SP, Kimmelman J. Are outcome-adaptive allocation trials ethical? Clin Trials 2015;12(2):102-6

19 van der Lee JH, Wesseling J, Tanck MWT, et al. Efficient ways exist to obtain the optimal sample size in clinical trials in rare diseases. J Clin Epidemiol 2008;61(4):324-30.

20 Bothwell LE, Kesselheim AS. The real-world ethics of Adaptive-Design clinical trials. Hastings Cent Rep 2017;47(6):27-37.

21 Sim J. Outcome-adaptive randomization in clinical trials: issues of participant welfare and autonomy. Theor Med Bioeth 2019;40(2):83-101.

22 Fleischman AR, Collogan K. Research with children. In: Emanuel EJ, ed. The Oxford textbook of clinical research ethics. Oxford University Press, 2008: 446-60.

23 Cerqueira FP, Jesus AMC, Cotrim MD. Adaptive design: a review of the technical, statistical, and regulatory aspects of implementation in a clinical trial. Ther Innov Regul Sci 2020;54(1):246-58

24 Kruger M. Promoting access to safe medicines for children: guest review. Current Allergy Clin Immunol 2017;30(1):12-14.

25 Fleischman AR. Ethical issues in neonatal research involving human subjects. Semin Perinatol 2016;40(4):247-53.

26 Bavdekar S. Pediatric clinical trials. Perspect Clin Res 2013:4(1):89.

27 Yan D, Wages NA, Dressler EV. Improved adaptive randomization strategies for a seamless phase I//I dose-finding design. J Biopharm Stat 2019;29(2):333-47.

28 Chevret S. Bayesian adaptive clinical trials: a dream for statisticians only? Stat Med 2012;31(11-12):1002-13.

29 Modi N, Vohra J, Preston J, et al. Guidance on clinical research involving infants, children and young people: an update for researchers and research ethics committees. Arch Dis Child 2014;99(10):887-91.

30 Meurer WJ, Legocki L, Mawocha S, et al. Attitudes and opinions regarding confirmatory adaptive clinical trials: a mixed methods analysis from the adaptive designs accelerating promising trials into treatments (ADAPT-IT) project. Trials 2016:17(1):373.

31 Gore R, Chugh PK, Tripathi CD, et al. Pediatric off-label and unlicensed drug use and its implications. Curr Clin Pharmacol 2017;12(1):18-25.

32 Sharav VH. Children in clinical research: a conflict of moral values. The American Journal of Bioethics 2003;3(1):12-59.
33 Emanuel EJ, Wendler D, Grady C. An Ethical Framework for Biomedical Research.. In: Emanuel EJ, ed. The Oxford textbook of clinical research ethics. Oxford University Press, 2008: 123-35.

34 Afolabi MOS. Resolving the identity dilemmas of Western healthcare in Africa: towards ethical and pragmatic approaches. Culture and Dialogue 2020;8(1):147-65.

35 Baud O, Maury L, Lebail F, et al. Effect of early low-dose hydrocortisone on survival without bronchopulmonary dysplasia in extremely preterm infants (PREMILOC): a double-blind, placebo-controlled, multicentre, randomised trial. Lancet 2016:387(10030):1827-36.

36 Zurynski Y, Deverell M, Dalkeith T, et al. Australian children living with rare diseases: experiences of diagnosis and perceived consequences of diagnostic delays. Orphanet $J$ Rare Dis 2017;12(1):1-9.

37 Eccleston C, Fisher E, Howard RF, et al. Delivering transformative action in paediatric pain: a Lancet Child \& Adolescent Health Commission. Lancet Child Adolesc Health 2021;5(1):47-87

38 Beauchamp TL, Childress JF. Principles of biomedical ethics. 7th ed. Oxford: Oxford University Press, 2013.

39 Park JJ, Thorlund K, Mills EJ. Critical concepts in adaptive clinical trials. Clin Epidemiol 2018; 10:343.

40 Katz AL, Webb SA, COMMITTEE ON BIOETHICS. Informed consent in decision-making in pediatric practice. Pediatrics 2016;138(2):e20161485

41 Montreuil M, Fortin J, Racine E. Children's assent within clinical care: A concept analysis. J Child Health Care 2020;1367493520976300.

42 Wendler DS. Assent in paediatric research: theoretical and practical considerations. J Med Ethics 2006:32(4):229-34.

43 Massetti T, Crocetta TB, Guarnieri R, et al. A didactic approach to presenting verbal and visual information to children participating in research protocols: the comic book informed assent. Clinics 2018;73:e207.

44 Weisleder P. Helping them decide: a scoping review of interventions used to help minors understand the concept and process of Assent. Front Pediatr 2020;8:25.

45 Wendler D. The Assent requirement in Pediatric Research. In: Emanuel EJ, Emanuel EJ, eds. The Oxford textbook of clinical research ethics. Oxford University Press, 2008: 661-9.

46 Popper KR. The open universe: an argument for indeterminism. Vol 2. Psychology Press, 1992.

47 Hansson S. The ethics of risk: ethical analysis in an uncertain world. Springer, 2013.

48 Tannert C, Elvers H-D, Jandrig B. The ethics of uncertainty. in the light of possible dangers, research becomes a moral duty. EMBO Rep 2007:8(10):892-6.

49 London AJ, systems Lhealth. Clinical equipoise and the ethics of response adaptive randomisation. J Medical Ethics 2018;44(6):409-15.

50 Posch M, Maurer W, Bretz F. Type I error rate control in adaptive designs for confirmatory clinical trials with treatment selection at interim. Pharm Stat 2011:10(2):96-104.

51 Giovagnoli A. The Bayesian design of adaptive clinical trials. Int J Environ Res Public Health 2021:18(2):530.

52 Villar SS, Robertson DS, Rosenberger WF. The Temptation of Overgeneralizing Response-Adaptive randomization. Clin Infect Dis 2020:ciaa1027.

53 Proschan M, Evans S. Resist the Temptation of Response-Adaptive randomization. Clin Infect Dis 2020;71(11):3002-4.

54 Chandereng T, Chappell R. How to do Response-adaptive randomization (RAR) if you really must. Clinical Infectious Dis 2020;4.

55 Wasserstein RL, Schirm AL, Lazar NA. Moving to a World Beyond " $p<0.05$ ". American Statistician 2019;73(1):1-9.

56 Wellek S. A critical evaluation of the current "p-value controversy". Biom J 2017;59(5):854-72.

57 Wasserstein RL, Lazar NA. ASA statement on statistical significance and P-values. In: Gruber CW, ed. The theory of statistics in psychology. Springer, Cham, 2020: pp. $1-10$.

58 Smith RJ. P> .05: The incorrect interpretation of "not significant" results is a significant problem. Am J Phys Anthropol 2020;172(4):521-7.

59 Ndebele P. Research Ethics. In: Chadwick R, ten Have H, Meslin EM, eds. The SAGE Handbook of healthcare ethics. Los Angeles: Sage Publishers, 2011: 306-25.

60 Laage T, Loewy JW, Menon S, et al. Ethical considerations in adaptive design clinical trials. Ther Innov Regul Sci 2017;51(2):190-9.

61 Berry DA. Adaptive clinical trials: the promise and the caution. J Clin Oncol 2011:29(6):606-9.

62 Brueckner M, Titman A, Jaki T, et al. Performance of different clinical trial designs to evaluate treatments during an epidemic. PLoS One 2018;13(9):e0203387.

63 Lasky T, Carleton B, Horton DB, et al. Real-World evidence to assess medication safety or effectiveness in children: systematic review. Drugs Real World Outcomes 2020;7(2):97-107

64 Schwemmer O. Cultural Morality and Moral Principles. In: Thiele F, Ashcroft RE, eds. Bioethics in a small world. Berlin: Springer, 2005: 5-16.

65 Afolabi MOS. A Vulnerability/solidarity framework for a global ethic: Historical \& contemporary applications. Revista Romana de Bioetica 2015;13:44-68.

66 ten Have HAMJ. Potter's Notion of Bioethics. Kennedy Inst Ethics J 2012;22(1):59-82.

67 Elder DP, Holm R, Kuentz M. Medicines for pediatric patients—biopharmaceutical, developmental, and regulatory considerations. J Pharm Sci 2017;106(4):950-60.

68 Kaye DK. The ethical Justification for inclusion of neonates in pragmatic randomized clinical trials for emergency newborn care. BMC Pediatr 2019;19(1):218. 
69 Levine RJ. The nature, scope, and justification of clinical research. In: Emanuel EJ, ed. The Oxford textbook of clinical research ethics. Oxford University Press, 2008: 211-21.

70 Afolabi MOS. Researching the vulnerables: issues of consent and ethical approval. Afr J Med Med Sci 2012;41 Suppl:7-11.

71 Gelfand SD. A partial defense of clinical equipoise. Res Ethics 2019;15(2):1-17.

72 Hey SP, London AJ, Weijer C. Is the concept of clinical equipoise still relevant to research? British Med J2017;359.

73 Veatch RM. The irrelevance of equipoise. J Med Philos 2007;32(2):167-83.

74 Miller FG. Is anything lost if we give up clinical equipoise? Clin Trials 2012;9(5):632.

75 Chau K, Koren G. The principle of equipoise in pediatric drug trials. Paediatr Drugs 2015;17(1):17-21.

76 Government of Canada. Tri-Council policy statement: ethical conduct for research involving Humans-TCPS 2 (2018), 2018. Available: https://ethics.gc.ca/eng/policypolitique_tcps2-eptc2_2018.html [Accessed April 22, 2021].
77 Cella M, Danhof M, Della Pasqua O. Adaptive trials in paediatric development: dealing with heterogeneity and uncertainty in pharmacokinetic differences in children. $\mathrm{Br} J$ Clin Pharmacol 2012;74(2):346-53.

78 Moss SE. Promoting ethical research practices: perspectives from pediatric populations. Semin Speech Lang 2011;32(4):289-97.

79 Tsang VWL, West L, Woods C, et al. Role of patients and parents in pediatric drug development. Ther Innov Regul Sci 2019;53(5):601-8.

80 Gill D, Crawley FP, LoGiudice M, et al. Guidelines for informed consent in biomedical research involving paediatric populations as research participants. Eur J Pediatr 2003; 162(7-8):455-8.

81 Dimairo M, Pallmann P, Wason J, et al. The Adaptive designs CONSORT Extension (ACE) statement: a checklist with explanation and elaboration guideline for reporting randomised trials that use an adaptive design. BMJ 2020;369:m115. 